\title{
APPLICATIONS OF TISSUE DOPPLER AND SPECKLE TRACKING ECHOCARDIOGRAPHY IN THE EARLY DETECTION OF DIASTOLIC DYSFUNCTION IN CANCER PATIENTS UNDERGOING ANTHRACYCLINE- BASED CHEMOTHERAPY
}

\author{
Chaithra Nayak1, Narasimha D. Pai' ${ }^{2}$ Padmakumar R ${ }^{3}$, Karthik S. Udupa ${ }^{4}$,Umesh Pai ${ }^{5}$, Francis N. P. Monteiro ${ }^{6}$
}

${ }_{1}^{1}$ Assistant Professor, Department of Cardiology, Kasturba Medical College (Affiliated to MAHE), Mangalore, Karnataka, India. ${ }^{2}$ Associate Professor, Department of Cardiology and Interventional Cardiology, Kasturba Medical College (Affiliated to MAHE), Mangalore, Karnataka, India.

${ }^{3}$ Professor, Department of Cardiology, Kasturba Medical College (Affiliated to MAHE), Manipal, Karnataka, India.

${ }^{4}$ Associate Professor and HOD, Department of Medical Oncology, Kasturba Medical College (Affiliated to MAHE), Manipal, Karnataka, India.

${ }^{5}$ Assistant Professor, Department of Cardiology, (Selection Grade), School of Allied Health Sciences, MAHE, Manipal, Karnataka, India. ${ }^{6}$ Professor, Department of Forensic Medicine and Toxicology, A. J. Institute of Medical Sciences (Affiliated to Rajiv Gandhi University of Health Sciences, Mangalore, Karnataka, India.

\section{BACKGROUND}

\section{ABSTRACT}

The preferred choice of treatment for malignant tumours is chemotherapy, which carries equal risk of cardiotoxicity. These drugs are dose dependent, hence worsen cardiac function from treatment completion to follow-up. Late effects of anthracyclines include subclinical cardiac abnormalities like systolic and diastolic dysfunction that are usually masked in the routine conventional echocardiography. With the regular use of tissue Doppler imaging, 2D derived strain and strain rate besides conventional Doppler techniques helps in early diagnosis of myocardial systolic and diastolic dysfunction, thereby reducing cardiac disease progression.

\section{MATERIALS AND METHODS}

A prospective cohort study was employed to establish diastolic dysfunction in cancer patients undergoing anthracycline-based chemotherapy in the Department of Cardiology, Kasturba Medical College (Affiliated to MAHE), Manipal. The study group consisted of patients with no past history of anticancer treatment, baseline left ventricular EF $>50 \%$ and normal levels of renal and hepatic enzymes. This study was performed on 190 patients, 70 males and 120 females with mean age of $38 \pm 16$ years. The subjects undergoing first and second dose of chemotherapy were named as first follow-up (F1) and second follow-up (F2). Among these, only 70 subjects could complete two follow-ups of chemotherapy. Parameters of conventional echocardiography were recorded and integrated with TDI parameters such as LVEF, transmitral E and A velocity and TDI ( $\mathrm{e}^{\prime} / \mathrm{a}^{\prime}=$ ratio between early diastolic velocity of myocardium and late diastolic velocity a' and systolic myocardial velocity (Sm). LV systolic function in terms of EF was measured by modified biplane Simpson's method. Newer parameters like 2D derived strain and strain rate imaging were included that consisted of Peak Systolic Strain Rate (SSR), Early Diastolic Strain Rate (EDSR) and Late Diastolic Strain Rate (LDSR).

\section{RESULTS}

This study was performed on 190 patients, 70 males and 120 females with mean age of $38 \pm 16$ years. The subjects undergoing first and second dose of chemotherapy were named as first follow-up (F1) and second follow-up (F2). Among these, only 70 subjects could complete two follow-ups of chemotherapy. Among the conventional 2D echocardiographic parameters representing $\mathrm{LV}$ systolic and diastolic function assessed from the baseline to first follow-up showed no statistically significant changes. TDI and strain values were not found to be significant at the early doses of chemotherapy ( 4 weeks from the baseline), though an increase in systolic strain rate in anterior wall of LV $(-1.29 \pm 0.42$ vs $-1.8 \pm 0.78, p<0.001)$ was noted during first follow-up. TDI performed in the inter follow-up group was significantly reduced in basal IVS in terms of early myocardial tissue velocity $(0.09 \pm 0.02$ vs $0.07 \pm 0.02$ ). Pairwise comparison between baseline study and second follow-up (F2) showed statistically significant decrease in early diastolic strain rate in two segments of LV, LW $(1.58 \pm 0.49$ and $1.34 \pm 0.49, \mathrm{p}<0.001)$ and AS $(1.78 \pm 2.15$ and $1.33 \pm 0.45$, $\mathrm{p}<0.001)$. The inter follow-up group when compared for systolic strain rate showed a decline in systolic strain rate and early diastolic strain rate $(1.93 \pm 0.68$ and $1.34 \pm 0.49, \mathrm{p}<0.001)$.

\section{CONCLUSION}

Patients exposed to anthracycline treatment previously show subtle abnormalities (both systolic and diastolic), though the convention 2D echocardiography shows a normal ejection fraction. The cardiac function deteriorates over time leading to CHF which can be reduced by early diagnosis through newer Doppler independent techniques like strain and strain rate. Hence, 2D derived strain rate imaging with its ease of use and reproducibility over TDI should be utilised as potential non-invasive tool for the early detection of subclinical longitudinal myocardial abnormalities in chemotherapy patients.

\section{KEY WORDS}

Tissue Doppler Imaging, Congestive Heart Failure, Longitudinal Strain, Early Diastolic Strain Rate, Left Ventricular Ejection Fraction.

HOW TO CITE THIS ARTICLE: Nayak C, Pai ND, Padmakumar R, et al. Applications of tissue Doppler and speckle tracking echocardiography in the early detection of diastolic dysfunction in cancer patients undergoing anthracycline-based chemotherapy. J. Evolution Med. Dent. Sci. 2018;7(35):3816-3820, DOI: 10.14260/jemds/2018/856 


\section{BACKGROUND}

Anthracyclines derived drugs are preferred for different types of malignant tumours undergoing chemotherapy. These drugs being cardiotoxic comprises a wide range of cardiac effects like arrhythmias and minimal changes in blood pressure. The anthracyclines are strong cardiotoxic drugs; however, their use in clinical sector is reduced due to its harmful side effects. The risk of developing cardiac toxicity will increase with a cumulative dose of anthracycline. In our setup, the study focuses on the effect of anthracycline drugs on the cardiac systolic and diastolic function. Various noninvasive Doppler techniques have been used to assess the systolic and diastolic function among which TDI is more sensitive marker and highly reproducible. 2D derived strain and strain rate are the newer techniques that are load independent and better markers of tissue deformation and regional ventricular function compared to TDI. Strain and strain rate data provide prognostic information and thus can be utilised in detecting the early diastolic dysfunction in the chemotherapy group. The objective of the study was to evaluate early diastolic dysfunction in cancer patients receiving Anthracycline chemotherapy by using Tissue Doppler imaging.

\section{MATERIALS AND METHODS}

A prospective cohort study was employed to establish diastolic dysfunction in cancer patients undergoing anthracycline based chemotherapy in the Department of Cardiology, Kasturba Medical College, Manipal. The study group included patients who had no history of anticancer treatment, baseline M-mode derived LVEF > 50\% and normal levels of renal and hepatic enzymes. Patients with an existing heart disease, previous exposure to mediastinal irradiation/chemotherapy with anthracycline, use of other concomitant agents known to have cardiotoxic effects and absence of important pathologies were excluded from the study.

A prospective cohort study was employed at Department of Cardiology, KMC Manipal (Affiliated to MAHE), to establish diastolic dysfunction in cancer patients undergoing anthracycline based chemotherapy.

The study was performed with Transthoracic Echocardiography using Vivid 7-echocardiography system (GE) with a $2.5 \mathrm{MHz}$ transducer. The cardiac parameters were assessed by conventional Doppler echocardiography which included chamber dimensions, LVEF, transmitral Doppler flow velocities. Parameters of conventional echocardiography were recorded and integrated with TDI parameters such as LVEF, transmitral E and A velocity, and TDI $\left(\mathrm{e}^{\prime} / \mathrm{a}^{\prime}=\right.$ ratio between early diastolic velocity of myocardium and late diastolic velocity a' and systolic myocardial velocity (Sm). LV systolic function in terms of EF was measured by modified

'Financial or Other Competing Interest': None.

Submission 03-07-2018, Peer Review 08-08-2018,

Acceptance 14-08-2018, Published 27-08-2018.

Corresponding Author:

Dr. Narasimha D. Pai,

Associate Professor,

Department of Cardiology and Interventional Cardiology,

Kasturba Medical College, Ambedkar Circle, (Affiliated to MAHE),

Mangalore, Karnataka, India.

E-mail: narasimhapai@yahoo.com

DOI: $10.14260 /$ jemds $/ 2018 / 856$ biplane Simpson method. Newer parameters like 2D derived strain and strain rate were included that consisted Peak Systolic Strain Rate (SSR), Early Diastolic Strain Rate (EDSR) and Late Diastolic Strain Rate (LDSR).

The sample was decided as per the convenience. It included 190 patients, 70 males and 120 females with mean age of $38 \pm 16$ years. The subjects undergoing first and second dose of chemotherapy were named as first follow-up (F1) and second follow-up (F2). Among these, only 70 subjects could complete two follow-ups of chemotherapy.

All the data were entered in the excel sheet and analysed by SPSS 16.0 software. Descriptive statistics were mentioned as mean and standard deviation. Paired ' $\mathrm{t}$ ' test was carried out to test the significant difference between baseline and follow-ups.

\section{RESULTS}

This study was performed in 190 patients, 70 (37\%) males and $120(63 \%)$ females with mean age of $38 \pm 16$ years. The subjects undergoing first and second dose of chemotherapy were named as first follow-up (F1) and second follow-up (F2). Among these, only 70 subjects could complete two follow-ups of chemotherapy. These 70 subjects, 25 (36\%) males and $45(64 \%)$ females required anthracycline therapy for Breast cancer $(n=25)$, Large B-cell lymphoma $(n=10)$, Acute lymphoblastic leukaemia $(n=10)$, Ca metastatic ovary $(\mathrm{n}=5)$, Leiomyosarcoma $(\mathrm{n}=5)$, Acute myeloblastic leukaemia $(n=5)$, plasma cell myeloma $(n=5)$ and Acute promyelocytic leukaemia $(n=5)$.

\begin{tabular}{|c|c|c|c|c|}
\cline { 2 - 5 } \multicolumn{1}{c|}{} & Baseline & Follow-up 1 & Follow-up 2 \\
\hline \multirow{2}{*}{$\begin{array}{c}\text { Total no. of } \\
\text { patients }\end{array}$} & $\mathrm{n}=70$ & $\mathrm{n}=70$ & $\mathrm{n}=70$ \\
\hline \multirow{2}{*}{ Gender } & Male & $26(37 \%)$ & $25(36 \%)$ & $25(36 \%)$ \\
\cline { 2 - 5 } & Female & $45(63 \%)$ & $45(64 \%)$ & $45(64 \%)$ \\
\hline \multicolumn{2}{|c|}{ Table 1. Gender } \\
\hline \multicolumn{2}{|c|}{ Distribution- Total }
\end{tabular}

Table 1. Gender Distribution- Total

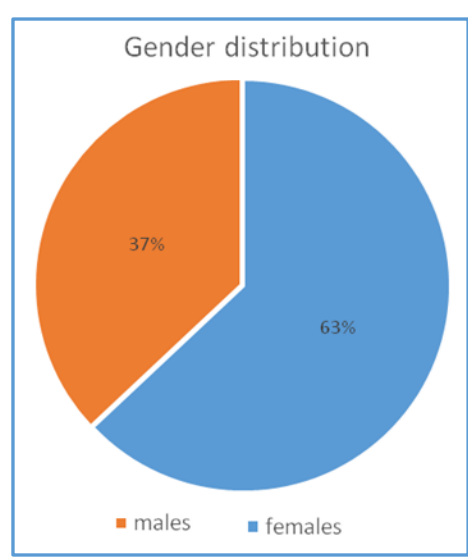

No significant changes were found in 2D derived strain values from baseline to first follow-up (Table 2).

\begin{tabular}{|c|c|c|c|}
\hline LV Segments & Baseline & F2 & P value \\
\hline IVS & $-20 \pm 4.06$ & $-20.42 \pm 3.28$ & NS \\
\hline LW & $-19.41 \pm 5.28$ & $-21.38 \pm 3.67$ & NS \\
\hline IW & $-18.75 \pm 4.27$ & $-22.66 \pm 4.65$ & NS \\
\hline AW & $-18.41 \pm 4.48$ & $-20.07 \pm 4.47$ & NS \\
\hline PW & $-18.05 \pm 4.3$ & $-18.27 \pm 2.56$ & NS \\
\hline AS & $-18.54 \pm 2.7$ & $-21.11 \pm 4.56$ & NS \\
\hline Table 2. Comparison of 2D derived strain values between \\
Baseline and Second Follow-up \\
\hline \multicolumn{4}{|c}{} \\
\hline
\end{tabular}


LV- Left Ventricle; IVS- Interventricular Septum; LWLateral Wall; IW- Inferior Wall; AW- Anterior Wall; PWPosterior Wall; AS- Anteroseptum; NS- Not Significant; F2Second follow-up.

No significant changes were noted in systolic strain rate from baseline to second follow-up (Table 3).

\begin{tabular}{|c|c|c|c|}
\hline LV Segments & Baseline & F2 & P value \\
\hline IVS & $-1.32 \pm 0.56$ & $-1.24 \pm 0.26$ & NS \\
\hline LW & $-1.24 \pm 0.49$ & $-1.34 \pm 0.25$ & NS \\
\hline IW & $-1.26 \pm 0.39$ & $-1.52 \pm 0.46$ & NS \\
\hline AW & $-1.29 \pm 0.42$ & $-1.38 \pm 0.26$ & NS \\
\hline PW & $-1.51 \pm 0.72$ & $-1.42 \pm 0.37$ & NS \\
\hline AS & $-1.33 \pm 0.45$ & $-2.51 \pm 0.84$ & NS \\
\hline \multicolumn{4}{|c|}{ Table 3. Comparison of systolic strain rate between } \\
Baseline and the Second Follow-up \\
\hline
\end{tabular}

LV- Left Ventricle; IVS- Interventricular Septum; LWLateral Wall; IW- Inferior Wall; AW- Anterior Wall; PWPosterior Wall; AS- Anteroseptum; NS- Not Significant; F1First follow-up.

On comparison of the baseline parameters to second follow-up, the conventional echocardiographic parameters representing systolic and diastolic function of LV showed statistically significant increase in End Diastolic Volume (EDV) $\mathrm{mL}(58.86 \pm 13.26$ vs $67.43 \pm 13.74, \mathrm{p}<0.037$ ) (Table-4).

\begin{tabular}{|c|c|c|c|}
\hline LV Parameters & Baseline & F2 & P value \\
\hline $\begin{array}{l}\text { End Diastolic Diameter } \\
\text { (EDD) }\end{array}$ & $43.57 \pm 4.05$ & $\begin{array}{c}46.71 \pm \\
3.97\end{array}$ & NS \\
\hline End Systolic Diameter (ESD) & $26.93 \pm 3.05$ & $\begin{array}{c}29.29 \pm \\
3.92\end{array}$ & NS \\
\hline Ejection fraction (\%) & $68.79 \pm 3.26$ & $\begin{array}{c}66.79 \pm \\
4.24\end{array}$ & NS \\
\hline $\begin{array}{l}\text { End Diastolic Volume (EDV) } \\
\mathrm{mL}\end{array}$ & $58.86 \pm 13.26$ & $\begin{array}{c}67.43 \pm \\
13.74\end{array}$ & 0.037 \\
\hline $\begin{array}{l}\text { End Systolic Volume (ESV) } \\
\text { mL }\end{array}$ & $\begin{array}{c}19.64 \pm \\
4.98\end{array}$ & $\begin{array}{c}23.21 \pm \\
5.98\end{array}$ & NS \\
\hline $\begin{array}{l}\text { Maximal velocity of early } \\
\text { diastolic filling (E) }(\mathrm{m} / \mathrm{s})\end{array}$ & $\begin{array}{c}0.77 \pm \\
.21\end{array}$ & $\begin{array}{c}0.82 \pm \\
0.19\end{array}$ & NS \\
\hline $\begin{array}{c}\text { Maximal velocity of late } \\
\text { diastolic filling }(\mathrm{A})(\mathrm{cm} / \mathrm{S})\end{array}$ & $0.76 \pm 0.23$ & $\begin{array}{c}0.67 \pm \\
0.19\end{array}$ & NS \\
\hline $\mathrm{E} / \mathrm{A}$ & $1.22 \pm 0.79$ & $\begin{array}{c}1.26 \pm \\
0.37\end{array}$ & NS \\
\hline $\mathrm{LA}(\mathrm{mm})$ & $27.5 \pm 6.84$ & $\begin{array}{c}26.45 \pm \\
6.37\end{array}$ & NS \\
\hline $\mathrm{AO}(\mathrm{mm})$ & $25.36 \pm 4.48$ & $\begin{array}{c}26.14 \pm \\
4.78\end{array}$ & NS \\
\hline LA/AO & $1.09 \pm 0.16$ & $1.1 \pm 0.2$ & NS \\
\hline
\end{tabular}

LV- Left Ventricle; IVS- Interventricular Septum; LWLateral Wall; IW- Inferior Wall; AW- Anterior Wall; PWPosterior Wall; AS- Anteroseptum; NS- Not Significant; F1First follow-up.
TDI and strain values were not found to be significant at the early doses of chemotherapy ( 4 weeks from the baseline). However, a significant increase in systolic strain rate in anterior wall of $\operatorname{LV}(-1.29 \pm 0.42$ vs $-1.8 \pm 0.78, \mathrm{p}<0.001)$ was noted during first follow-up (Table 5).

\begin{tabular}{|c|c|c|c|}
\hline LV Segments & Baseline & F1 & P value \\
\hline IVS & $-1.32 \pm 0.56$ & $-1.38 \pm 0.33$ & NS \\
\hline LW & $-1.24 \pm 0.49$ & $-1.42 \pm 0.4$ & NS \\
\hline IW & $-1.26 \pm 0.39$ & $-1.41 \pm 0.39$ & NS \\
\hline AW & $-1.29 \pm 0.42$ & $-1.8 \pm 0.78$ & $<0.001$ \\
\hline PW & $-1.51 \pm 0.72$ & $-1.51 \pm 0.72$ & NS \\
\hline AS & $-1.33 \pm 0.45$ & $-1.44 \pm 0.42$ & NS \\
\hline
\end{tabular}

Table 5. Comparison of Systolic Strain Rate between the

Baseline Study and First Follow-Up of Chemotherapy

LV- Left Ventricle; IVS- Interventricular Septum; LWLateral Wall; IW- Inferior Wall; AW- Anterior Wall; PWPosterior Wall; AS- Anteroseptum; NS- Not Significant; F1First follow-up.

We also noted a statistically significant reduction in the early diastolic strain rate of AS segment during the first follow-up from baseline study $(1.78 \pm 2.15$ vs $1.65 \pm 0.58$, $\mathrm{p}<0.001$ ) (Table 6).

LV- Left Ventricle; IVS- Interventricular Septum; LWLateral Wall; IW- Inferior Wall; AW- Anterior Wall; PWPosterior Wall; AS- Anteroseptum; NS- Not Significant; F1First follow-up.

Pairwise comparison between baseline study and second follow-up (F2) showed statistically significant decrease in early diastolic strain rate in two segments of $L V$, LW $(1.58 \pm 0.49$ and $1.34 \pm 0.49, \mathrm{p}<0.001)$ and AS $(1.78 \pm 2.15$ and $1.33 \pm 0.45, \mathrm{p}<0.001$ ) (Table 6).

\begin{tabular}{|c|c|c|c|}
\hline LV Segments & Baseline & F2 & P value \\
\hline IVS & $1.49 \pm 0.46$ & $1.55 \pm 0.49$ & $\mathrm{NS}$ \\
\hline LW & $1.58 \pm 0.49$ & $1.34 \pm 0.49$ & $<0.001$ \\
\hline IW & $1.62 \pm 0.38$ & $1.84 \pm 0.68$ & $\mathrm{NS}$ \\
\hline AW & $1.68 \pm 0.72$ & $2.55 \pm 2.68$ & $\mathrm{NS}$ \\
\hline PW & $1.82 \pm 0.42$ & $1.41 \pm 0.54$ & $\mathrm{NS}$ \\
\hline AS & $1.78 \pm 2.15$ & $1.33 \pm 0.45$ & $<0.001$ \\
\hline \multicolumn{4}{|c|}{ Table 6. Comparison of Early Diastolic Strain Rate between } \\
the Baseline Study and Second Follow-up \\
\hline
\end{tabular}

LV- Left Ventricle; IVS- Interventricular Septum; LWLateral Wall; IW- Inferior Wall; AW- Anterior Wall; PWPosterior Wall; AS- Anteroseptum; NS- Not Significant; F2Follow-up 2.

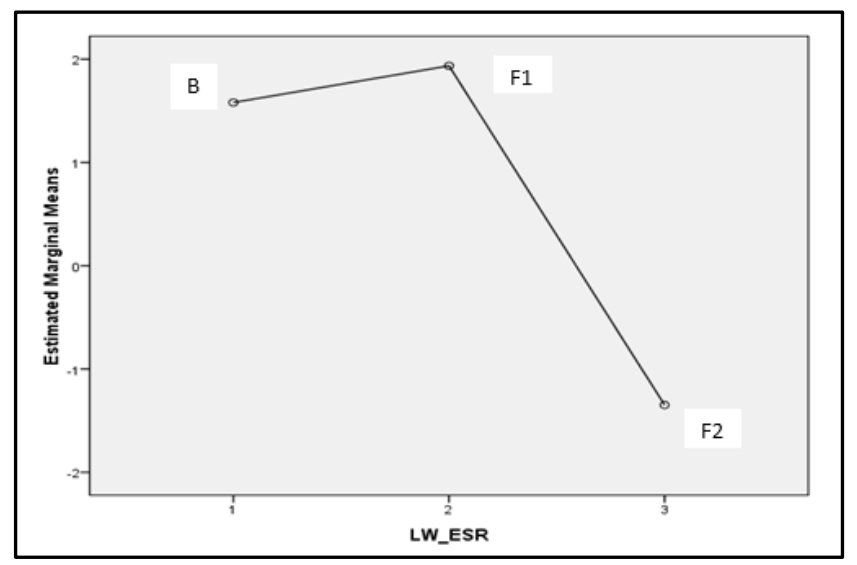


The global early diastolic strain rate in the chemotherapy group initially increased till the first chemo dose and declined rapidly after the higher dose of chemotherapy.

The conventional 2D echocardiographic parameters showed no statistical differences. The inter follow-up group also showed statistical significant reduction in systolic strain rate $(1.8 \pm 0.78$ vs $1.38 \pm 0.26, \mathrm{p}<0.001)$ in basal AW segment of LV (Table 7).

\begin{tabular}{|c|c|c|c|}
\hline LV Segments & F1 & F2 & P value \\
\hline IVS & $-1.38 \pm 0.33$ & $1.24 \pm 0.26$ & NS \\
\hline LW & $-1.42 \pm 0.4$ & $1.34 \pm 0.25$ & NS \\
\hline IW & $-1.41 \pm 0.39$ & $1.52 \pm 0.46$ & NS \\
\hline AW & $1.8 \pm 0.78$ & $1.38 \pm 0.26$ & $<0.001$ \\
\hline PW & $-1.51 \pm 0.72$ & $1.42 \pm 0.37$ & NS \\
\hline AS & $-1.44 \pm 0.42$ & $2.51 \pm 0.84$ & NS \\
\hline \multicolumn{4}{|c|}{ Table 7. Comparison of Systolic Strain Rate between First } \\
and Second follow-up of Chemotherapy \\
\hline
\end{tabular}

LV- Left Ventricle; IVS- Interventricular Septum; LWLateral Wall; IW- Inferior Wall; AW- Anterior Wall; PWPosterior Wall; AS- Anteroseptum; NS- Not Significant; F2follow-up 2.

Also, the early diastolic strain rate was reduced in the inter follow-up period $(1.93 \pm 0.68$ and $1.34 \pm 0.49$, $p<0.001)$.

\begin{tabular}{|c|c|c|c|}
\hline LV Segments & F1 & F2 & P value \\
\hline IVS & $1.75 \pm 0.49$ & $1.55 \pm 0.49$ & NS \\
\hline LW & $1.93 \pm 0.68$ & $1.34 \pm 0.49$ & $<0.001$ \\
\hline IW & $1.63 \pm 0.67$ & $1.84 \pm 0.68$ & NS \\
\hline AW & $1.8 \pm 0.78$ & $2.55 \pm 2.68$ & NS \\
\hline PW & $1.82 \pm 0.42$ & $1.41 \pm 0.54$ & NS \\
\hline AS & $1.65 \pm 0.58$ & $1.33 \pm 0.45$ & NS \\
\hline Table 8. Comparison of Diastolic Strain Rate between First \\
and second follow-up of Chemotherapy \\
\hline
\end{tabular}

No statistical significance was noted from baseline to first, second follow-up and inter follow-up period in terms of conventional Doppler parameters like E/A ratio, E/E' also $\mathrm{E}^{\prime} / \mathrm{A}^{\prime}$.

\section{DISCUSSION}

It is asserted by the research conducted by Steinherz et al, patients who have irregular echocardiograms at the end of their therapy are at the higher risk cardiomyopathy. Study of Steinherz et al revealed that after the completion of chemotherapy during the year, $12 \%$ of patients with a normal echocardiogram had abnormal echocardiograms during the follow-up. ${ }^{1}$ Dose of doxorubicin greater than $550 \mathrm{mg} / \mathrm{m}^{2}$, increases the incidence of congestive heart failure rapidly. This dose of doxorubicin recognised as an empiric limiting dose for doxorubicin-induced cardiotoxicity. ${ }^{2}$ In our study, 2D Echocardiographic parameter EDD (End Diastolic Dimension) showed no statistically significant reduction of dimensions within the subjects, while there were no changes observed in pairwise comparison. Other 2D echo parameters (ESD, EF, FS) did not show any changes throughout the study. Research conducted by the Valagussa et al showed that marker of the global LV function to be the TDI velocity profile of the mitral annulus, while velocity profiles found in left ventricular myocardial segments reflect regional myocardial function. ${ }^{3}$ Since Doppler orientation, radial left ventricular (LV) shortening and relaxation are better reflected by the TDI profile of the posterior left ventricular wall, while the lateral left ventricular (LV) wall and mitral annulus offer information on the longitudinal left ventricular function (LV). ${ }^{3}$ Though, the study by Valagussa $P$ et al showed that the TDI velocity profile of the mitral annulus is usually considered as a marker of the global LV function, ${ }^{3}$ our study was able to demonstrate early changes in the diastolic function in terms of $2 \mathrm{D}$ derived strain rate velocities that considers regional myocardial function when compared with TDI. The tissue Doppler imaging had also demonstrated early changes in diastolic function in the inter follow-up group with statistical significant value of $p<0.025$ as the chemo dose and the duration increased. In our data transmitral $\mathrm{E}$ and $\mathrm{A}$ velocities, E/A ratios did not significantly reduce during the study period. A 2D speckle tracking derived strain has overcome these limitations, which allows quantification of myocardial deformation in three dimensions, necessitating only B-mode images of standard echocardiographic examination. Its simplicity of use and reproducibility renders it a potentially positive method for detecting subclinical myocardial abnormalities in the long-term follow-up of chemotherapy patients. The 2D speckle tracking derived strain imaging performed in our study showed statistically significant difference in the global longitudinal strain imaging of IW $p<0.043$ in the chemo group, while the inter follow-up period comparison did not show much difference. The causes for this varied pattern of strain lessening have not been elucidated, but may be correlated to dissimilarity in regional wall stress, or to local variation in activation of signalling pathways implicated in apoptosis or fibrosis. ${ }^{4}$ Research conducted by Ganame et $\mathrm{al}^{4}$ on cohort of 56 children who had received anthracycline treatment up to 15 years also established regional decrease in TDI-derived longitudinal strain in the interventricular septum and LV lateral wall.

Progressive deterioration of longitudinal fibre dysfunction with an increasing severity of symptoms was elucidated by a 2D Speckle tracking echocardiography research on hypertensive patients. ${ }^{5}$ In contrast to it, radial and circumferential strain were maintained in less advanced stages of heart failure. However, finding in other hypertensive cohorts, highlighted a supranormal radial function in these subjects.6,7 This suggests that radial and circumferential contractility may compensate for early longitudinal dysfunction, thus initially maintaining normal overall LV function. In our study, the 2D speckle tracking imaging showed a statistically significant reduction of peak systolic longitudinal strain rate (SSR) in the AW segment of LV with $\mathrm{p}<0.05$ as well the inter follow-up comparison showed significant reduction in systolic strain rate with $\mathrm{p}<0.001$. The 2D speckle tracking derived Early Diastolic Strain Rate (EDSR) showed significant reduction in lateral wall, posterior wall and anteroseptum segments of LV with $\mathrm{p}<0.001, \mathrm{p}<0.022$ and $\mathrm{p}<0.001$ respectively. From the baseline study, early changes were noted in the lateral and anteroseptal segments of LV after the first and second doses of chemotherapy in terms of early diastolic strain rate, except for posterior wall which did not show significant changes in inter follow-up, but overall showed a significant reduction in strain rate $(\mathrm{p}<0.02)$. The late diastolic strain rate parameter (LDSR) did not show any significant difference in the chemotherapy group as well inter follow-up group. Hence, 
conventional 2D echocardiographic parameters is less sensitive for the early detection of preclinical cardiac disease (subclinical). Moreover, it is influenced by contractility and preload/ afterload effects leading to transient changes. Considering the systolic strain rate parameter as a marker of systolic function, we in our study were able to show a linear increase in the longitudinal systolic strain rate among the chemotherapy group that initially increased till first chemo dose and gradually declined linearly after higher dose. Therefore, other measurements of systolic function and diastolic function should be used to detect early cardiotoxicity in addition to LVEF. ${ }^{8,9}$ The study performed by Daniel Di Lisi et al showed E'/A' ratio measured with the tissue Doppler was more sensitive than E/A ratio at baseline and after chemotherapy. ${ }^{10}$ In our study E'/A' parameter was found to be significant in the chemotherapy group. The E/A ratio (Doppler parameter of diastolic function) did not show significant differences in the chemotherapy group as well in inter follow-ups. No changes were found in the early diastolic velocity (E), early systolic velocity (A), left atrium, aortic dimensions and LA/Ao ratio. On the basis of our results in the chemotherapy group and in agreement with the literature, anthracyclines are more cardiotoxic and their cardiotoxicity is dose dependent. As per the obtained results and considering the entire patient population, anti-cancer drugs causes alterations in systolic and diastolic functions and 2D derived longitudinal strain rate is more sensitive than TDI and conventional echocardiography in assessing the LV diastolic function in early doses of chemotherapy. Also the subclinical changes in systolic function can be identified easily with longitudinal systolic strain rate. In effect, asymptomatic alteration of systolic and diastolic functions were shown by $2 \mathrm{D}$ derived strain rate imaging very much early than TDI and 2D speckle tracking imaging, but not conventional echocardiography in the early months after starting chemotherapy.

\section{CONCLUSION}

- $\quad$ Patients exposed to anthracycline treatment previously show subtle abnormalities (both systolic and diastolic), though the conventional 2D echocardiography show a normal ejection fraction.

- The diagnosis of subclinical cardiac disease is of much importance in patients undergoing anthracycline therapy and hence tissue Doppler techniques play an important role along with conventional echocardiography.

- Early the diagnosis, better is the survival in the setting of diastolic dysfunction induced by anthracyclines.

- The cardiac function deteriorates over time leading to $\mathrm{CHF}$, which can be reduced by early diagnosis through newer Doppler independent techniques like strain and strain rate.
- Impairment in the cardiac function needs early detection for adjustment of the drug dose and to preserve the ventricular compliance.

- Systolic function is determined by the conventional Mmode techniques, whereas strain rate could show reduction in the systolic values from the initial treatment to final despite having a normal LVEF.

- In our setup, 2D derived strain rate could detect early changes in diastolic dysfunction within one follow-up of chemotherapy compared to TDI.

- 2D derived strain rate imaging with its ease of use and reproducibility over TDI is a potential non-invasive tool for the early detection of subclinical longitudinal myocardial abnormalities in these patients.

\section{REFERENCES}

[1] Steinherz LJ, Steinherz PG, Tan CT, et al. Cardiac toxicity 4 to 20 years after completing anthracycline therapy. JAMA 1991;266(12):1672-7.

[2] Shan K, Lincoff M, Young JB. Anthracycline-induced cardiotoxicity. Ann Intern Med 1996;125(1):47-58.

[3] Valagussa P, Zambetti M, Biasi S, et al. Cardiac effects following adjuvant chemotherapy and breast irradiation in operable breast cancer. Ann Oncol 1994;5(3):209-16.

[4] Ganame J, Claus P, Uyttebroeck A, et al. Myocardial dysfunction late after low-dose anthracycline treatment in asymptomatic paediatric patients. J Am Society of Echocardiography 2007;20(12):1351-8.

[5] Waggoner AD, Bierig SM. Tissue Doppler imaging: a useful echocardiographic method for the cardiac sonographer to assess systolic and diastolic ventricular function. J Am Soc Echocardiogr 2001;14(12):1143-52.

[6] Palmon LC, Reichek N, Yeon SB, et al. Intramural myocardial shortening in hypertensive left ventricular hypertrophy with normal pump function. Circulation 1994;89(1):122-31.

[7] Aurigemma GP, Silver KH, Priest MA, et al. Geometric changes allow normal ejection fraction despite depressed myocardial shortening in hypertensive left ventricular hypertrophy. J Am College of Cardiology 1995;26(1):195-202.

[8] Jurcut R, Wildiers H, Ganame J, et al. Detection and monitoring of cardiotoxicity - What does modern cardiology offer? Support Care Cancer 2008;16(5):43745.

[9] Ganz WI, Sridhar KS, Ganz SS, et al. Review of tests for monitoring doxorubicin-induced cardiomyopathy. Oncology 1996;53(6):461-70.

[10] Di Lisi D, Bonura F, Macaione F, et al. Chemotherapy induced cardiotoxicity: role of Tissue Doppler in the early diagnosis of left ventricular dysfunction. AntiCancer drugs 2011;22(5):468-72. 